\title{
Consciência do Direito ao Atendimento Odontológico entre Usuários de uma Clínica de Ensino
}

\section{Awareness Regarding the Right to Dental Care among Users of a Teaching Clinic}

\author{
Elaine Toledo Pitanga Fernandes \\ Doutoranda em Odontologia. Professora do Curso de Odontologia \\ da Universidade Vale do Rio Doce. \\ Endereço: Rua Osvaldo Cruz, 302/901, Centro, CEP 35010-210, Go- \\ vernador Valadares, MG, Brasil. \\ E-mail: elainepitangaœdatapoint.inf.br \\ Efigênia Ferreira e Ferreira \\ Doutora em Ciência Animal. Professora associada da Faculdade de \\ Odontologia da Universidade Federal de Minas Gerais. \\ Endereço: Rua Rio Negro, 1260/30I, Grajaú, CEP 30430-400, Belo \\ Horizonte, MG, Brasil. \\ E-mail: efigeniafळgmail.com
}

\section{Resumo}

Este estudo teve por objetivo identificar o conhecimento dos usuários de uma clínica de ensino quanto aos direitos básicos relativos às possibilidades de atendimento odontológico. Utilizou-se como instrumento, entrevista estruturada, realizada com 56 usuários (estimativa de proporção). Observou-se que o ingresso nesse serviço de saúde é bem distante do que se poderia definir como consciência cidadã. Os usuários conseguem o atendimento por amizade $(32,1 \%)$ ou através da emergência (28,6\%), quando não conseguem, a alternativa mais apontada passa a ser a clínica privada $(42,8 \%)$, sinalizando que o direito constitucional à saúde não é incorporado pela população deste estudo, que tenta solucionar seus problemas de maneira individual e com recursos próprios. Mesmo em casos de emergência (dor), percebe-se a continuação dessa postura (50\%). Apenas quatro indivíduos buscaram atendimento no setor público, o que reforça o desconhecimento do direito básico ao atendimento em saúde e das outras alternativas que o serviço organizado oferece. A caracterização de que um serviço dessa natureza se constitui em uma benesse é confirmada quando $76,8 \%$ dos entrevistados declararam desconhecer quem financia seu tratamento e $42,8 \%$ não fariam reclamações, apesar de insatisfeitos com o serviço. Apesar do trabalho de esclarecimento realizado pela instituição junto aos usuários, ainda se observa a falta de informação sobre direito e cidadania, fator que impede a participação política. A comunidade entrevistada não apresenta características de grupo consciente dos direitos relativos ao atendimento odontológico.

Palavras-chave: Participação comunitária; Saúde bucal. 


\section{Abstract}

The aim of this study was to identify the knowledge users of a teaching clinic have regarding basic rights related to the possibilities of dental care. A structured interview was conducted with 56 users (proportion estimate). Upon entering the healthcare service, users are far from what could be described as aware citizens. They obtain care through friendship (32.1\%) or cases of urgency $(28.6 \%)$. When they do not obtain care, the most mentioned alternative is a private clinic (42.8\%), which indicates that the constitutional right to healthcare is not incorporated by the population of the present study, as they attempt to solve their problems individually and with their own resources. This line of thinking remains (50\%) even in cases of urgency (pain), as only four individuals reported they would seek care in the public sector. This underlines the lack of knowledge of the basic right to healthcare and other alternatives that the organized public clinic offers. The characterization that a service of this nature performs favors is confirmed, as $76.8 \%$ of the interviewees reported not knowing who finances their treatment and $42.8 \%$ would not file complaints despite being dissatisfied with the service. In spite of the explanatory work carried out by the institution, users still do not have information about their rights and citizenship, a factor that prevents political participation. The interviewed community does not display the characteristics of a group that is aware of their rights regarding dental care. Keywords: Community Participation; Oral Health.

\section{Introdução}

A Carta de Ottawa, documento resultante da Primeira Conferência Internacional de Promoção da Saúde, define promoção de saúde como o processo de capacitação da comunidade para atuar na melhoria de sua qualidade de vida e saúde, incluindo maior participação no controle desse processo (World Health Organization, 1986).

O reforço à participação comunitária continuou em pauta nas discussões internacionais subsequentes em Adelaide, Austrália, em 1988 e Jacarta, Indonésia, em 1997 (World Health Organization, 1997).

A promoção da saúde prevê um processo a partir do qual os indivíduos e as comunidades estariam em condições de exercer maior controle sobre sua vida, aumentando assim as chances de serem mais saudáveis (Nutbean, 1996). Segundo Buss (2000), é essencial para as iniciativas da promoção da saúde o incremento do poder técnico e político das comunidades (empowerment) na fixação de prioridades e tomada de decisões e na definição e implementação de estratégias para alcançar melhor nível de saúde.

Por isso, a participação dos usuários nos serviços públicos de saúde vem sendo amplamente discutida, considerando ser esse um fator fundamental na qualidade desejada para os serviços. A identificação de problemas e soluções relacionadas às suas demandas contribuirão para ações de saúde mais efetivas (Andrade e Vaitsman, 2002), desenvolvendo nos usuários responsabilidade e o direito, ou seja, a cidadania.

Uma comunidade usuária de um serviço tem o empowerment quando ela, e não somente o profissional, estabelece também as prioridades em saúde, de forma sensível e participativa. Frequentemente, essa é uma ideia de difícil aceitação para profissionais ou gestores de saúde ( Málaga e Ramírez, 2001), visto que eles foram treinados em um modelo no qual o "provedor" de cuidados em saúde é o expert e o usuário, o recipiente de informação e de cuidado (Portilho e Paes, 200o).

Mais do que repassar informações e induzir determinados comportamentos, o empowerment deve buscar apoiar pessoas e coletivos a realizarem suas próprias análises para que tomem as decisões que 
considerarem corretas, desenvolvendo a consciência crítica e a capacidade de intervenção sobre a realidade (Carvalho, 2004).

Novos modos de se fazer saúde devem incorporar como diretriz uma postura que encare os usuários na sua singularidade de sujeitos portadores de direitos, em substituição a uma perspectiva que entende os usuários como suplicantes e beneficiários dos serviços (Carvalho, 2004).

Mas como o usuário se percebe portador do direito à saúde? A partir da década de 1980, a sociedade brasileira sofreu mudanças com relação à consciência de seu direito à saúde. A saúde como condição necessária à vida digna tem despertado a todos, incluindo aqueles que estão à margem do mercado consumidor. No entanto, a expressão do direito, incluindo o direito à saúde, varia conforme a consciência individual que depende da organização social predominante (Dallari, 1988).

Tudo se inicia com a visão que cada um tem do mundo. Segundo Roso (2007), cada indivíduo a constrói ao longo da vida representando como ele vê, interpreta e percebe os fatos. Apesar de subjetiva, ela depende também das relações estabelecidas com o mundo, o que a autora nomina subjetividade social. Essa visão do mundo pode delimitar o que a pessoa considera certo ou errado, bom ou mal, mas na maioria das vezes essa reflexão não ocorre. A consciência sobre essas práticas é uma construção ao mesmo tempo individual e social e pode determinar o comportamento do indivíduo.

A respeito do serviço de saúde, o usuário muitas vezes delimita o acesso à sua condição socioeconômica e considera de certa maneira natural que a oferta de serviços públicos seja dependente da capacidade do Estado. Percebe e aceita que o serviço é preparado para determinadas queixas e as suas podem não estar incluídas neste rol (Assis e col., 2003). $\mathrm{O}$ direito à saúde fica condicionado à existência ou não de recurso - o seu próprio ou o público.

Lefèvre e colaboradores (2002) ressaltam um dos grandes conflitos atuais que tem de um lado o direito à saúde, garantido por lei, e de outro, o mercado que condiciona a saúde ao poder de compra.

O próprio serviço de saúde incrementa esse conflito, quando passa a ser seletivo e excludente, já que muitas vezes não oferece o que o usuário necessita. Exemplo claro dessa afirmação pode ser observado nas baixas taxas de comparecimento de idosos a serviços odontológicos nas avaliações de acesso e uso dos serviços. No ultimo Levantamento Nacional de Saúde Bucal, dos idosos examinados $65,69 \%$ relataram não consultar o dentista há mais de 3 anos, sendo a dor o motivo da consulta para $48,12 \%$ dos que procuraram (Brasil, 2004). Considerando o percentual de desdentados entre os idosos brasileiros e a não oferta de prótese pelos serviços, eles podem não estar vendo motivos para procurar $o$ atendimento.

Uma questão importante no processo de construção da consciência política é a ressocialização contínua, construída durante as práticas da vida, que surgem, cotidianamente, segundo as oportunidades disponíveis para cada sujeito. Essa questão pode ser observada e construída mediante práticas como o diálogo e a escuta (Silva, 2007).

Nesse sentido, investir em ações que favoreçam o empowerment, pode resultar em processos de mobilização sociais, inaugurando ações coletivas para mudança social, promovendo formação da consciência do direito. Mas, segundo Prado (2002), o processo de mudança inclui a necessidade de três aspectos fundamentais: o processo de identificação coletiva, a passagem das relações de subordinação para a consciência da condição de opressão e a delimitação de fronteiras políticas entre grupos sociais.

Desse modo, nas instituições de atenção à saúde, a participação do usuário deveria contemplar maior democratização da informação; reconhecimento dos usuários, por parte dos profissionais, como sujeitos no processo do cuidado com a saúde e não somente como objetos de práticas e prescrições; conscientização dos usuários quanto aos seus direitos e ao seu papel na defesa dos próprios interesses (Andrade e Vaitsman, 2002).

Para iniciar estudos nessa linha, considerando não terem sido identificados na literatura artigos com essa abordagem, o objetivo deste trabalho foi identificar entre usuários de uma clínica de ensino seu conhecimento sobre direitos básicos, relacionados às possibilidades do atendimento odontológico. 


\section{Metodologia}

Trata-se de um estudo de abordagem quantitativa e de caráter exploratório descritivo, cuja população foi constituída por usuários de uma clínica integrada de ensino, de uma instituição prestadora de serviços odontológicos pelo Sistema Único de Saúde (SUS), que atende por demanda espontânea a comunidade do município e região. Em função do caráter didático, essas clínicas devem conjugar o ensino com o serviço de atenção à saúde, oferecendo aos alunos um meio de vivenciar a prática profissional através do atendimento à comunidade, exercitando assim a relação entre a teoria e a prática. Constituem-se em espaços privilegiados não só para o aprendizado de procedimentos técnicos, mas também para o exercício da reflexão ética dos futuros profissionais (Gonçalves e Verdi, 2007).

A Secretaria de Pacientes da instituição é responsável pela operacionalização do atendimento, caracterizando-se por isso como a porta de entrada desses serviços. Os usuários são cadastrados pela secretaria em duas categorias: pacientes regulares (aqueles que conseguiram vagas para realizar tratamento odontológico em sua totalidade) ou pacientes de urgência (aqueles que apresentam sintomatologia dolorosa e/ou outras necessidades que demandam atendimento imediato).

Foram incluídos como voluntários neste estudo usuários com idades iguais ou superiores a 18 anos, de ambos os sexos.

Para o cálculo da amostra, utilizou-se o método de estimativa de proporção, considerando-se o padrão de 50\%, nível de significância de 9o\%, e erro de 10\% (Snedecor e Cochran, 1989). Após a correção final da amostra, com base no universo finito de 280 usuários (média de usuários atendidos por semestre) obteve-se um $n$ final de 56 usuários. Os dados foram coletados a partir de uma entrevista estruturada, que continha questões abertas relacionadas ao direito ao atendimento odontológico. As entrevistas aconteceram na área de espera das clínicas e foram realizadas por quatro acadêmicos do último período de Odontologia, devidamente calibrados para a função.

Os usuários entrevistados foram selecionados por conveniência, isto é, aqueles que estavam pre- sentes no momento das entrevistas e se dispuseram a participar do estudo.

Após a realização das entrevistas, os resultados foram analisados e categorizados segundo a convergência das respostas, estabelecendo-se os seguintes temas para discussão: acesso ao atendimento odontológico, financiamento do atendimento odontológico e direito ao atendimento odontológico. As frequências das respostas foram computadas em percentuais.

Os participantes assinaram um termo de consentimento livre e esclarecido, assegurando o caráter confidencial de suas respostas e seu direito de não identificação, salientando a voluntariedade da participação. Este estudo foi aprovado pelo Comitê de Ética em Pesquisa da Instituição pesquisada (Parecer $n^{0}$ 18/2005).

\section{Resultados e Discussão}

Os resultados são apresentados de acordo com os temas observados após a análise das entrevistas.

Inicialmente, com relação ao acesso, $100 \%$ dos entrevistados relataram ter o conhecimento do processo para conseguir uma vaga para tratamento nas clínicas da instituição. Entretanto, analisando os fatores por eles apresentados para conseguir a vaga, (Tabela 1) percebe-se que eles desconsideram a lógica dos critérios de acesso estabelecidos pela instituição. Os usuários têm seus critérios próprios, sendo apontados com maior frequência: "ter um conhecido na instituição" (32,1\%) e "ser atendido como urgência" (28,6\%).

\section{Tabela I - Distribuição do $\mathrm{n}^{\circ}$ de entrevistados segundo as alternativas de acesso ao tratamento nas Clinicas de uma instituição de ensino odontológico, Minas Gerais, 2007}

\begin{tabular}{lcc} 
Alternativas & $n$ & $\%$ \\
Através de um conhecido na instituição & 18 & 32,1 \\
Através da emergência/urgência & 16 & 28,6 \\
Encaminhamentos externos informais & 8 & 14,3 \\
Inscrição & 7 & 12,5 \\
Informações de outros pacientes & 5 & 8,9 \\
Outros & 2 & 3,6 \\
\hline TOTAL & 56 & 100 \\
\hline
\end{tabular}


Pode-se afirmar que na ausência, ou mesmo no não reconhecimento de um sistema formalmente organizado, os usuários, diante da necessidade de tratamento, tentam "se virar" para conseguir a vaga. Inexiste nesses casos a decisão tomada corretamente, resultante de uma consciência crítica e capacidade de intervenção sobre a realidade (Carvalho, 2004).

Percebe-se também que, diante da crença na incapacidade de conseguir por si mesmo uma vaga para tratamento, o usuário busca ajuda com algum conhecido. A relação que se estabelece é a de favor prestado, tornando explícita a falta de poder e de consciência do direito. Para Gonçalves e Verdi (2007), o privilégio do atendimento através de amigos, conhecidos ou parentes caracteriza um abuso da autoridade de quem concede o "favor", desrespeitando simultaneamente a autonomia dos que conseguiram e dos que aguardam por atendimento.

Outra estratégia utilizada pelos usuários é a queixa de dor como motivo da consulta. As queixas, segundo Marques e Lima (2007), são construídas social e historicamente, conforme as relações vividas. Embora sem entender a lógica oficial dos serviços, o usuário percebe que dor é prioritária e torna mais rápida a solução do problema por ele apresentado. Além do atendimento à queixa apresentada, o usuário antevê a possibilidade de continuidade do tratamento.

Questionados sobre a atitude que tomariam diante da ausência de vagas para tratamento odontológico na instituição, os entrevistados apresentaram como alternativa (Tabela 2) em $1^{\mathrm{a}}$ opção, a clínica particular com utilização de recursos próprios $(42,8 \%)$ e, em $2^{\text {a }}$ opção, outro local sem dispêndio próprio (17,8\%).

O direito constitucional garantido de acesso ao atendimento não é incorporado pela população deste estudo, considerando que quase $50 \%$ dela considerou que não podendo realizar o tratamento nessa instituição teriam de solucionar o problema individualmente, da maneira que puderem com recursos próprios.

\section{Tabela 2 - Distribuição do $n^{\circ}$ de entrevistados segundo as alternativas quando não se consegue 0 acesso ao tratamento nas Clinicas de uma instituição de ensino odontológico, Minas Gerais, 2007}

\begin{tabular}{lcc} 
Alternativas & $n$ & $\%$ \\
Clínica privada/recursos próprios & 24 & 42,8 \\
Outro local sem dispêndio próprio/ & 10 & 17,8 \\
posto de saúde & & \\
Aguardar vaga na Univale & 9 & 16,1 \\
Desistir/conformar-se com a situação & 9 & 16,1 \\
Outros & 4 & 7,2 \\
\hline TOTAL & 56 & 100 \\
\hline
\end{tabular}

Assim, como na grande maioria das instituições, as normas impostas pelo serviço fogem ao entendimento do usuário, sobretudo pelo autoritarismo como são estabelecidas. Determina-se o que pode e o que não pode ser feito, cabendo ao usuário acatar sem discussão, atitudes contrárias ao empowerment que busca (Málaga e Ramírez, 2001). As alternativas conseguidas pelos usuários são determinadas pela criatividade, que tenta contornar as decisões do "provedor", do expert (Portilho e Paes, 200o).

Quando o problema é "dor de dente", um percentual de $75 \%$ dos usuários indicaram a Univale - centro de referencia para o atendimento de urgência - como o local a ser procurado nesse tipo de situação, desconhecendo outros locais oferecidos pelo serviço público municipal. Dos usuários que disseram buscar a instituição do estudo para o problema de dor (Tabela 3) 50\% relataram que procurariam uma clínica privada caso não conseguissem ser atendidos. Embora a dor seja um fator que motiva muito o indivíduo a buscar uma solução, 23,8\% dos indivíduos se conformariam com a situação (aguardar, desistir), ou tentariam solucionar o problema através da automedicação (16,7\%). Apenas quatro indivíduos buscariam atendimento no setor público, o que reforça o desconhecimento das outras alternativas que o serviço organizado oferece, direito básico relacionado ao atendimento em saúde. 
Alem da conscientização do direito, a população necessita da informação do que lhe é garantido (Dallari, 1988).

\section{Tabela 3 - Distribuição do $n^{\circ}$ de entrevistados segundo as alternativas para o fato de não se conseguir acesso ao atendimento de emergência, nas Clinicas de uma instituição de ensino odontológico, Minas Gerais, 2007}

\begin{tabular}{lcc} 
Alternativas & $n$ & $\%$ \\
Clínica privada/recursos próprios & 21 & 50,0 \\
$\begin{array}{l}\text { Outro local sem dispêndio próprio/ } \\
\text { posto de saúde }\end{array}$ & 4 & 9,5 \\
Aguardar vaga na Univale & 6 & 14,3 \\
Desistir/conformar com a situação & 4 & 9,5 \\
Automedicação & 7 & 16,7 \\
\hline TOTAL & 42 & 100 \\
\hline
\end{tabular}

O Artigo 196 da Constituição Federal Brasileira de 1988 assegura que "a saúde é um direito de todo cidadão brasileiro e um dever do estado" (Brasil, 1988) inclusive com destinação de verbas específicas para isso. No entanto, $76,8 \%$ dos entrevistados relataram desconhecer quem financia o tratamento odontológico recebido por eles na instituição.

Esse dado indica que os usuários ainda não são capazes de perceber que o tratamento recebido é um direito garantido por lei. Ressalta-se o caráter de gratuidade que, segundo Gonçalves e Verdi (2007), é muitas vezes atribuído pelos próprios professores, que desconsideram o que já foi previamente pago pela população. $O$ atendimento é então incluído no rol de "favor ou caridade". Se o usuário não entende quem financia o tratamento e não tem consciência do direito expresso na Constituição, fica convencido de que realmente foi contemplado com um "favor".

A desinformação por parte dos usuários merece uma reflexão especial. Na instituição em estudo, eles recebem, no início do tratamento, um folder explicativo, contendo instruções sobre o tratamento, esclarecimentos sobre o convênio com o SUS e-outras informações que a pesquisa mostrou não terem sido apreendidas. Evidenciou-se que não tem sido eficaz o meio escolhido para veicular as orientações relativas ao serviço. Longe de ser uma estratégia “empoderadora”, pode estar se configurando como um instrumento de poder, um manual de ordens a serem cumpridas (Carvalho e Gastaldo, 2008).

Andrade e Vaitsman (2002) consideram que a dificuldade dos usuários para reconhecer o serviço como um direito e não um favor se dê em função da relação de desigualdade econômica, social e cultural em que se eles se encontram.

A análise dos dados remete-nos à importância do reforço da ação comunitária, um dos campos de ação definidos pela Carta de Otawa (World Health Organization, 1986) para a Promoção da Saúde. O primeiro requisito para a capacidade de mobilização de uma comunidade, através de seus membros, a fim de chegar ao empowerment, deveria ser o conhecimento de seus direitos e deveres (Buss, 200o), partindo da democratização da informação (Andrade e Vaitsman, 2002).

Com relação ao conhecimento de outros locais na cidade, além da instituição, que ofereceria atendimento "de graça", 55,3\% dos usuários responderam não ter conhecimento de outro local do município ao qual pudessem recorrer. Um percentual de 44,7\% citou unidades de saúde do Sistema Público Municipal como outra alternativa para o referido atendimento.

Esses dados refletem a desinformação da população estudada no que diz respeito às possibilidades de atendimento odontológico que deveriam ser oferecidas pelo município, podendo tal fato representar o reflexo de uma divulgação inadequada dos serviços por parte dos gestores de saúde.

Outra observação que pode ser feita é que, apesar de $44,7 \%$ dos entrevistados relatarem saber da existência das unidades de saúde do sistema público, quando perguntados quem o procurariam em caso de dor, somente $14,3 \%$ se lembraram que esses locais existem (Tabela 3). Questionados a quem recorreriam para atendimento em casos de dor se não conseguissem atendimento nas clínicas da instituição do estudo, somente $7,1 \%$ deles citaram as unidades de saúde. Isso pode significar que apesar de se saber que a unidade de saúde existe, ela não está incluída na relação de direitos garantidos, incorporados pela população - quando há necessidade, a população não se lembra que ela existe.

O empowerment é um dos processos exigidos para a formação do cidadão-sujeito. Vale refletir, no 
momento, se a universidade estará contribuindo de fato para a formação desse sujeito, para a transformação da sociedade, uma de suas missões, através do desenvolvimento crítico dos indivíduos, permitindo sua melhor inserção nos espaços coletivos (Carvalho, 2004).

Com relação à possibilidade de reclamar do atendimento recebido nas clínicas da instituição, 32 (57,2\%) usuários relataram que fariam uma reclamação caso não ficassem satisfeitos com o atendimento recebido. Dos 24 (42,8\%) indivíduos que relataram que não reclamariam, apesar de insatisfeitos com o serviço, 16 não o fariam por medo de "perder a vaga" e quatro por ser gratuito o atendimento: "de graça até injeção na testa”.

A percepção de que é um tratamento gratuito, um favor recebido, coloca o indivíduo em posição inferior na relação estabelecida, completamente vulnerável, sem capacidade para se autorregular, sem possibilidade de dizer sim quando quer dizer sim e não quando quer dizer não.

\section{Considerações Finais}

Através dos dados obtidos, percebe-se que os informantes que compõem uma parcela representativa dos usuários que frequentam a clínica de ensino do estudo não apresentam características que poderiam classificá-los como parte de uma comunidade consciente dos direitos relativos ao atendimento odontológico.

O envolvimento da clientela dos serviços de saúde em graus que ultrapassam a mera utilização passiva é algo desejável. A avaliação compartilhada junto aos usuários poderá ser um instrumento para a mudança das práticas de saúde nas clínicas, de maneira democrática, com controle social e participação comunitária preconizada pela reforma sanitária brasileira.

O reconhecimento da saúde como direito social e, consequentemente, dever do estado, pode possibilitar o exercício pleno do direito à saúde pelos cidadãos brasileiros. No entanto, falta à população informações para isso.

A falta de informação é um fator que impede a participação política, portanto, deveria ser um passo inicial do processo, a divulgação das informações para a população, de forma respeitosa e construtiva. Sua ausência pode levar a população a não ter condições de se mobilizar para reivindicar seus direitos, tendo por isso dificuldades para lutar por eles. Para instrumentalizar a população, objetivando o exercício de sua cidadania plena, é preciso contar com a educação, com as instituições de ensino

Conclui-se que a instituição deve repensar sua prática, de maneira que se processe a efetiva transformação desejada, tendo como caminho, necessariamente a ser percorrido, o estabelecimento de estratégias que contribuam de fato para a formação do cidadão-sujeito, bem como para o fortalecimento do poder da comunidade.

\section{Agradecimentos}

Nossos agradecimentos a Renata Pimenta Giacomini, Gisele Silva Brito, Polyana Argolo Souza e Patrick Magno Martins, acadêmicos do $8^{\circ}$ período do Curso de Odontologia da FACS-Univale pela colaboração na coleta dos dados utilizados neste trabalho.

\section{Referências}

ANDRADE, G. R. B.; VAISTMAN, J. Apoio social e redes: conectando solidariedade e saúde, Ciência \& saúde coletiva, Rio de janeiro, v. 7, n. 4, p. 925-934, 2002.

ASSIS, M. M. A.; VILLA, T. C. S.; NASCIMENTO, M. A. A. Acesso aos serviços de saúde: uma possibilidade a ser construída na prática, Ciência \& saúde coletiva, Rio de Janeiro, v.8, n.3, p.815-823, 2003.

BRASIL. Constituição da República Federativa do Brasil, 1988. Disponível em:<http://www.planalto. gov.br/ccivil_03/constituicao/constitui\%C3\%A7ao. htm> Acesso em: 10 abril 2009.

BRASIL. Ministério da Saúde. Secretaria de Atenção à Saúde. Departamento de Atenção à Saúde. Coordenação Nacional de Saúde Bucal. Projeto SB Brasil 2003; Condições de saúde bucal da população brasileira 2002-2003 - resultados principais. Brasília: Coordenação Nacional de Saúde Bucal; 2004. 52 p. 
BUSS, P. M. Promoção da Saúde e qualidade de vida, Ciência \& saúde coletiva, Rio de Janeiro, v. 5, n.1, p.163-177, 2000.

CARVALHO, S. R. Os múltiplos sentidos da categoria "empowerment" no projeto de Promoção à Saúde. Cadernos de Saúde Pública, Rio de Janeiro, v. 20, n. 4, p.1088-1095, jul.-ago. 2004.

CARVALHO, S. R.; GASTALDO, D. Promoção à saúde e empoderamento: uma reflexão a partir das perspectivas critico-social e pós-estrutural. Ciência \& saúde coletiva, Rio de Janeiro, v. 13, suppl.2, p. 2029-2040, dec. 2008.

DALLARI, S. G. O direito à saúde, Revista de Saúde Pública, São Paulo, v. 22, n. 1, p. 56-63, 1988.

GONÇALVES, E. R.; VERDI, M. I. M. Os problemas éticos no atendimento a pacientes na clínica odontológica de ensino. Ciência \& saúde coletiva, Rio de Janeiro, v.12, n. 3, p.755-764, maio-jun. 2007.

LEFÈVRE, A. M. C. et al. Assistência publica à saude no Brasil: estudo de seis ancoragens. Saúde e Sociedade, São Paulo, v. 11, n. 2, p. 35-47, ago.-dez. 2002

MÁLAGA, H.; RAMÍREZ, M. L. C. Cómo empoderar a los excluídos em el nivel local. In: MÁLAGA, H.; RESTREPO, H. E. Promoción de la salud: cómo construir vida saludable. Bogotá: Panamericana, 2001. p.120-37.

MARQUES, G. Q.; LIMA, M. A. D. S. Demandas de usuários a um serviço de pronto atendimento e seu acolhimento ao sistema de saúde. Revista Latino-Americana de Enfermagem, Ribeirão Preto, v. 15, n. 1, p. 13-19, 2007.

Recebido em: 13/08/2009

Reapresentado em: 11/04/2010

Aprovado em: 10/06/2010
NUTBEAN, D. Glosario de Promoción de la Salud. In: Organización Panamericana de la Salud. Promocion de la salud: una antologia. Washington: D.C.,OPS, 1996. p. 383-403.

PORTILHO, J. A. C.; PAES, A. M. C. Autopercepção de qualidade de vida relativa à saúde bucal. Revista Brasileira de Odontologia em Saúde Coletiva, Brasília, v. 1, n. 1, p. 75-88, 2000.

PRADO, M. A. M. Da mobilidade social à constituição da identidade política: reflexões em torno dos aspectos psicossociais das ações coletivas. Psicologia em revista, Belo Horizonte, v. 8, n. 11, p.59-71, jun. 2002.

ROSO, A. Psicologia social da saúde: tornamonos eternamente responsáveis por aqueles que cativamos. Aletheia, Canoas, n. 26, p. 80-94, 2007.

SILVA, A. S. A identificação de adversários, de sentimentos antagônicos e de (in)eficácia política na formação da consciência política no MST Paulista. Estudos e Pesquisas em Psicologia, Rio de Janeiro, v. 7, n. 1, p. 105-126, abr. 2007.

SNEDECOR, G. W.; COCHRAN, W. G. Statistical methods. 8. ed. Ames: Iowa State Universit: 1989.

WORLD HEALTH ORGANIZATION. 1986. Carta de Ottawa. In: BRASIL. Ministério da Saúde. Secretaria de Políticas de Saúde. Promoção da Saúde: declaração de Alma-Ata: Carta de Ottawa: declaração de Adelaide: declaração de Sundsvall: declaração de Santafé de Bogotá: declaração de Jacarta: rede dos Megapaíses: declaração do México. Brasília: Ministério da Saúde/ FIOCRUZ, 2001. p. $11-18$.

WORLD HEALTH ORGANIZATION. 1997. Declaración de Jakarta. In: BUSS, P. M. (ed.) 1998. Promoção da Saúde e Saúde Pública. Rio de Janeiro: Escola Nacional de Saúde Pública, [s.d.]. p. $174-178$. 